\title{
A Pellet 3D Printer: Device Design and Process Parameters Optimization
}

\author{
Shiyi Liu, ${ }^{1,2}$ Peng Zhao $\mathbb{D}^{1,2}$ Senyang Wu, ${ }^{1,2}$ Chengqian Zhang, ${ }^{1,2}$ Jianzhong Fu, ${ }^{1,2}$ \\ and Zichen Chen ${ }^{1,2}$ \\ ${ }^{1}$ The State Key Laboratory of Fluid Power and Mechatronic Systems, College of Mechanical Engineering, Zhejiang University, \\ Hangzhou 310027, China \\ ${ }^{2}$ Key Laboratory of $3 D$ Printing Process and Equipment of Zhejiang Province, College of Mechanical Engineering, \\ Zhejiang University, Hangzhou 310027, China
}

Correspondence should be addressed to Peng Zhao; pengzhao@zju.edu.cn

Received 10 August 2019; Accepted 4 October 2019; Published 28 November 2019

Academic Editor: Gyorgy Szekely

Copyright (c) 2019 Shiyi Liu et al. This is an open access article distributed under the Creative Commons Attribution License, which permits unrestricted use, distribution, and reproduction in any medium, provided the original work is properly cited.

\begin{abstract}
A novel pellet 3D printer was first developed, and its structure was constructed out of three main parts. The material used in this device was polycaprolactone (PCL), which was praised for its good characteristics in the biomanufacturing and chemical industries. Three essential parameters that had important effects on the diameter of the printed fibers were systematically studied using a $\mathrm{L}_{9}\left(3^{4}\right)$ orthogonal design table. Using the fused deposition modelling (FDM) method, some products were printed with this machine. Results showed that the stepper motor's speed had the most significant effect on the diameter of the printed fibers. The optimal parameters were, a stepper motor speed of $1.256 \mathrm{~mm}^{3} \mathrm{~s}^{-1}$, a nozzle moving speed of $9.6 \mathrm{~mm} \mathrm{~s}^{-1}$, and $1.1 \mathrm{~mm}$ of height between the nozzle and the platform. Defects like gaps, warping, and poor surface quality were found to be related to different combinations of process parameters. By using the developed pellet 3D printer, the pre-step of making filaments can be avoided, which will bring convenience to FDM 3D printing.
\end{abstract}

\section{Introduction}

Polycaprolactone (PCL) is widely used in tissue engineering involving engineering and natural science [1]. When the biological material and manufacturing method developed, its usage caught much attention [2-4]. PCL's good bio-compatibility and compatibility with other polymers, makes it a favorable material for scaffolds, especially in the biomanufacturing and chemical industries [5, 6]. Gao et al. fabricated nanofibrous PCL scaffolds with 3D controllable geometric shapes in order to carry cells and create an excellent environment for cell proliferation by means of electrospinning [7]. Didaskalou et al. introduced a sustainable membrane-based synthesis separation platform for enantioselective organocatalysis, and the make of the membrane makes the important component [8]. Wang et al. produced PCL/carbon nanotube scaffolds for bone applications by extrusion-additive manufacturing, and proved more beneficial advantages of PCL from chemical, physical, and biological points of view [9]. Xiao et al. used emulsion freeze-drying to prepare four types of porous scaffolds and investigated advantages and disadvantages of different weight ratios of $\mathrm{PCL} / \mathrm{PMCL}$ in order to sustain human induced hepatocytes [10]. Xue et al. combined PCL membranes with $\mathrm{Fe}_{3} \mathrm{O}_{4}$ nanoparticles via electrospinning technology, and showed its beneficial potential as a methylene blue catalyst with high recyclability [11]. In the view of the degrading characteristics of PCL, Ponjavic et al. introduced Poly-ethylene oxide (PEO) as the central or lateral segments in the PCL chain and investigated the copolymer hydrolysis and biodegradation properties [12]. Combining PCL with polylactic acid (PLA), Lin et al. improved the bond strength with a single-layer, temperature-adjusting transition method [13]. In general, PCL does play an important role in tissue engineering, biomaterials, and chemistry, however, it still requires suitable manufacturing processes, such as electrospinning and extrusion additive manufacturing.

As an additive manufacturing technique, $3 \mathrm{D}$ printing, that converts a digital model into a physical object, has features 
TABLE 1: Parameters of the customized screw.

\begin{tabular}{lcccccc}
\hline Parameter & External diameter & Helix angle & Screw ratio & Screw pitch & Width of groove & Depth of groove \\
Value & $\Phi 14 \mathrm{~mm}$ & $17^{\circ} 41^{\prime}$ & 4.57 & $5.3 \mathrm{~mm}$ & $4.00 \mathrm{~mm}$ & $3.2 \mathrm{~mm}$ \\
\hline
\end{tabular}

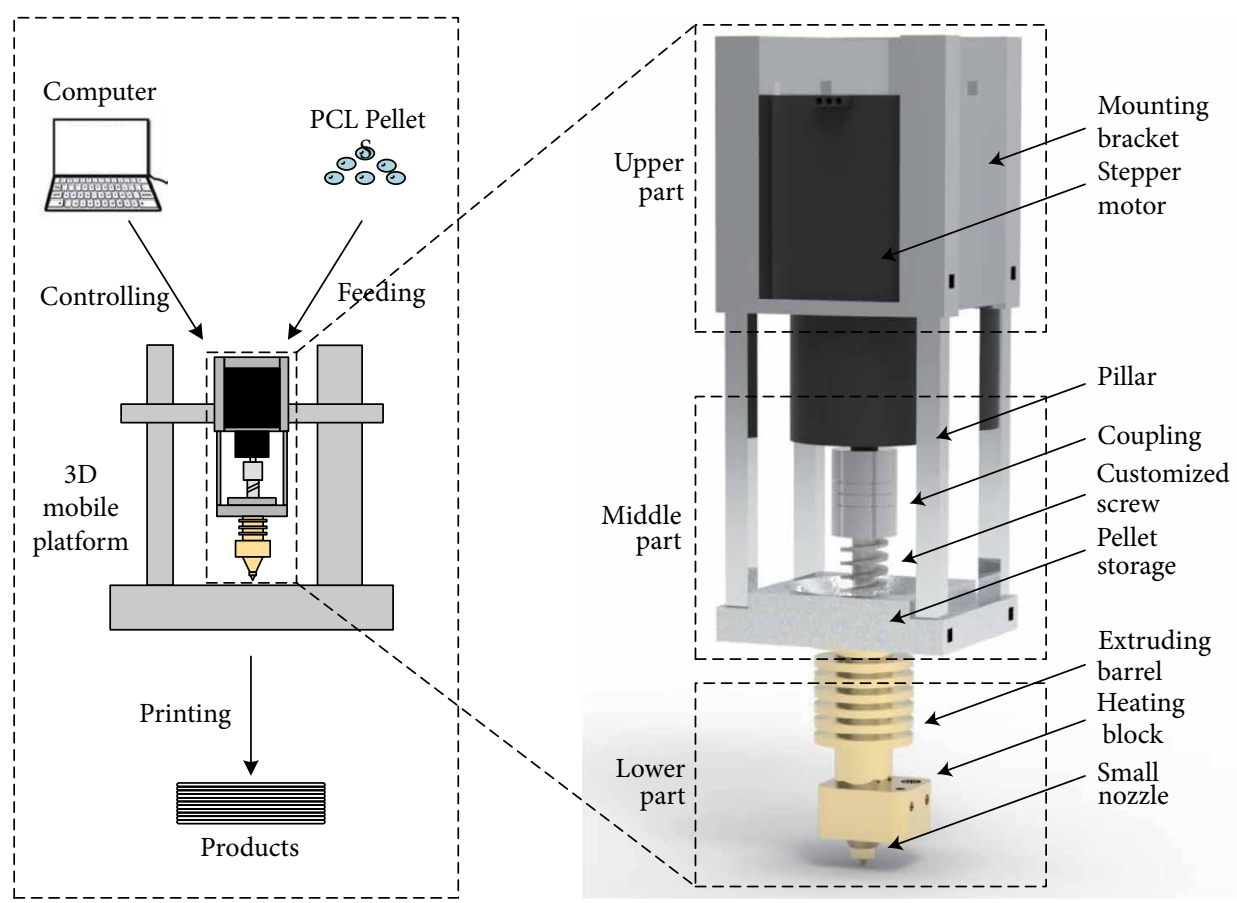

(a)

(b)

FIgURE 1: (a) The flow chart of the working system; (b) the details of the printer with three main parts.

prominently in fabricating functional materials and structures [14]. Selective laser melting (SLM), fused deposition modelling (FDM), and selective laser sintering (SLS) are three main technologies of $3 \mathrm{D}$ printing. With the process advantages of low cost, high speed, and convenience, FDM focuses on stacking a thermoplastic polymer, which is heated at the nozzle and extruded onto the previous layer or the platform, layer by layer. Typically, FDM can satisfy the requirements of various mechanical structures for diversified uses by combining different materials. For example, Kang et al. invented an integrated tissue-organ printer by using cell-laden hydrogels with biodegradable polymers (like PCL) to fabricate stable, humanscale tissue constructs of any shape [15]. Rao et al. investigated the potential of the close-looped recycling of PLA that used FDM [16]. Zhuang et al. used FDM in conjunction with PLA to prepare a series of plastic items that had anisotropic heat and resistance distributions [17]. Griffiths et al. made a design of experiments approach on the optimization of energy and waste during 3D printing, and proved positive results [18]. Furthermore, to improve the mechanical properties, some necessary parameters should be taken into consideration: layer thickness, width, orientation of filaments, and gap [19]. Yuk and Zhao improved the resolution of the printed fibers and printed thickened or curved patterns by optimizing the extruding speed, the moving speed of the nozzle, and the height of the nozzle above the platform [20]. However, inter-layer distortion, outer-layer warping, poor surface quality and weak mechanical properties still need to be solved [21, $22]$. Overcoming the difficulties of a limited number of thermoplastic materials is also a challenge that arises in FDM [19].

Many kinds of polymers have been used in 3D printing because they are easy to obtain, which is also valid for using polymers in FDM. PLA and acrylonitrile-butadiene-styrene copolymers (ABS) are commonly used polymers, which are usually produced as filaments in preparation for the next step. PCL's weak melt-strength means it cannot be extruded into filaments. Therefore, pure PCL always fails to meet the requirement of traditional FDM technology [3, 22]. Some biomaterials get bad influences from the step of making into filaments [23]. On the other hand, in comparison with filaments, plastic pellets have the advantages of lower cost, easier production process, and more freedom of size customization. However, there is little information about miniature pellet $3 \mathrm{D}$ printers. Goyanes et al. used powder materials to make drug products by $3 \mathrm{D}$ printing [24]. And Whyman et al. introduced a device for extruding PLA pellet polymers into product. Design of structure and temperature controlling system are mentioned [25]. Herein, we first developed a pellet 3D printer using pure PCL pellets for 3D printing. Then some parameters, such as the extruding speed, moving speed of the nozzle, and nozzle height, are optimized to get a better experience of the printed fibers through an orthogonal experiment using a $\mathrm{L}_{9}\left(3^{4}\right)$ 




FIGURE 2: The experiencing zones of pellets: (a) pellet zone; (b) glass transition zone; (c) melting zone.



FIGURE 3: The main process of printing: after feeding, PCL pellets were pre-heated and then printed layer by layer, as the product.

orthogonal design table. Moreover, the developed 3D printer can use more materials besides PCL, and its ability to print products more accurately and efficiently would become a reality with further advancements.

\section{Experiment}

2.1. Material. PCL $\left(\mathrm{Capa}^{\mathrm{TM}}\right.$ 6400) was purchased from Perstorp Co., (Shanghai, China), with a molecular weight of 37,000 , and melting temperature of $59^{\circ} \mathrm{C}$. The material was supplied in granular form, approx. $3 \mathrm{~mm}$ pellets. Its melt flow rate (MFR) ranged from 27 to $71 \mathrm{~g} / 10 \mathrm{~min}$.

2.2. Structure of Printer. Figure 1(a) showed the flow chart of the working system. The printer is shown in Figure 1(a) with three parts. The upper part consists of the stepper motor
TABLE 2: Factors and level of the orthogonal experiment.

\begin{tabular}{lcccc}
\hline & & \multicolumn{3}{c}{ Levels of each factor } \\
Factors & Parameters & 1 & 2 & 3 \\
\hline$A$ & $\begin{array}{c}\text { Speed of stepper motor } \\
\left(\mathrm{mm}^{3} \mathrm{~s}^{-1}\right)\end{array}$ & 5.814 & 3.681 & 1.256 \\
$B$ & $\begin{array}{c}\text { Moving speed of nozzle } \\
\left(\mathrm{mm} \mathrm{s}^{-1}\right)\end{array}$ & 6.4 & 8.0 & 9.6 \\
$D$ & $\begin{array}{c}\text { Height from nozzle to } \\
\text { platform }(\mathrm{mm})\end{array}$ & 0.9 & 1.1 & 1.3 \\
\hline
\end{tabular}

TABLE 3: Header design for the orthogonal experiment.

\begin{tabular}{lcccc}
\hline Factor & $A$ & $B$ & Blank & $C$ \\
Column & 1 & 2 & 3 & 4 \\
\hline
\end{tabular}

(model 42HB47PL020R-TK0) and the mounting bracket that is used to sustain the motor and connect the printer to the $3 \mathrm{D}$ mobile platform. The middle part is composed of the coupling, customized screw, pillars and pellet storage. The parameters of the customized screw are listed in Table 1. Considering the essential need that different pellets can be extruded by the device, the nonstandard screw is taken into account. The lower part contains the extruding barrel, the small nozzle $(\Phi=0.5 \mathrm{~mm})$ and the heating block. Except for the extruding barrel, the heating block and small nozzle are made of brass, while the bracket, pillars, and pellet storage are all aluminum. The small nozzle and barrel use threaded connections, while the other parts use pins to make the connection that are invisible in Figure 1(b).

2.3. Process of Experiment. Before extruding, PCL pellets were dried in a vacuum oven at $35^{\circ} \mathrm{C}$ for $12 \mathrm{~h}$. After feeding the PCL pellets into the storage, the stepper motor drove the screw to send pellets into the heating zone at an appropriate speed, then the temperature was set at $65^{\circ} \mathrm{C}$, which was a little higher than the melting temperature. As shown in Figure 2, the pellets went through the pellet zone, glass transition zone, and melting zone, then the molten PCL was extruded as fibers that were piled up layer by layer to form products like Figure 3 . The printing pattern depended on the G-code produced by a slicing software. And some input process parameters like line spacing $(0.6 \mathrm{~mm})$, and the filling rate $(100 \%)$ had been set appropriately to make the results of the experiment clearer.

When the temperature is set to be lower than melting temperature, PCL is hard to be extruded during glass transition period. If higher, PCL would gather at the exit of nozzle. The optimal range of temperature is narrow and the hysteresis of it has to be considered. To make the products printing more successful, the heating temperature was set aside temporarily and a further research would focus on it. In other words, the speed of stepper motor $C$, the moving speed of nozzle $V$, and the height from the nozzle to the platform $H$ would be the major factors that influenced the quality of the printed fibers. The speed of stepper motor $C$ was determined by the subdivided number of its motor driver, which could provide more than three levels of speed. The moving speed of nozzle $V$ and the height from the nozzle to the platform $H$ depended on the $3 \mathrm{D}$ 




(a)

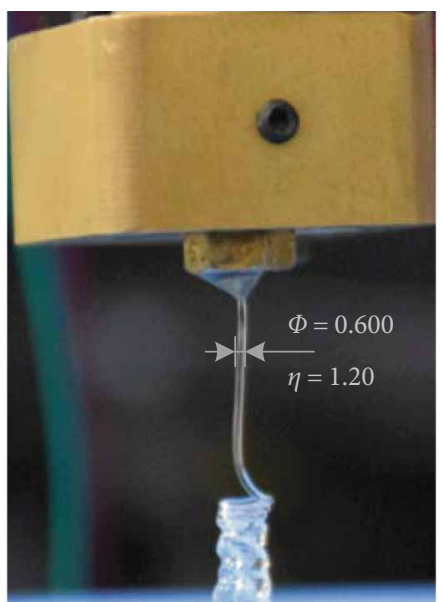

(b)

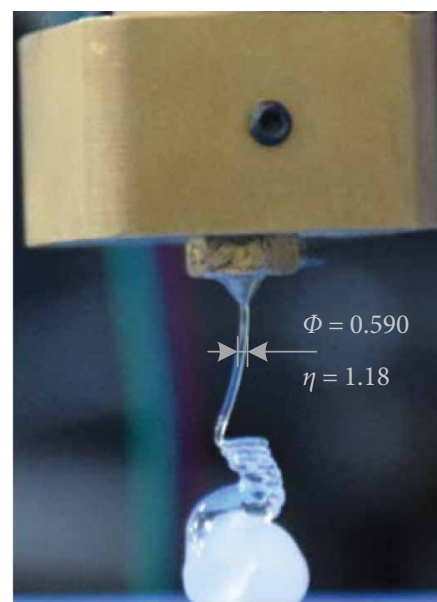

(c)

FIGURE 4: Different ratios of die swelling with the three speeds of the stepper motor: (a) $5.814 \mathrm{~mm}^{3} \mathrm{~s}^{-1}$; (b) $3.681 \mathrm{~mm}^{3} \mathrm{~s}^{-1}$; (c) $1.256 \mathrm{~mm}^{3} \mathrm{~s}^{-1}$.

TABLE 4: Result of the orthogonal experiment.

\begin{tabular}{lccccc}
\hline & \multicolumn{5}{c}{ Level and factor } \\
Experiment & $A$ & $B$ & Blank & $C$ & Diameter \\
\hline 1 & 1 & 1 & 1 & 1 & 1.77 \\
2 & 1 & 2 & 2 & 2 & 1.48 \\
3 & 1 & 3 & 3 & 3 & 1.54 \\
4 & 2 & 1 & 2 & 3 & 0.97 \\
5 & 2 & 2 & 3 & 1 & 1.04 \\
6 & 2 & 3 & 1 & 2 & 0.62 \\
7 & 3 & 1 & 3 & 2 & 0.62 \\
8 & 3 & 2 & 1 & 3 & 0.36 \\
9 & 3 & 3 & 1 & 1 & 0.41 \\
\hline
\end{tabular}

TABLE 5: Range analysis of the orthogonal experiment.

\begin{tabular}{lccc}
\hline Factor & $\begin{array}{c}\text { Speed of the } \\
\text { stepper motor }\end{array}$ & $\begin{array}{c}\text { Moving speed of } \\
\text { the nozzle }\end{array}$ & $\begin{array}{c}\text { Height from the } \\
\text { nozzle to the } \\
\text { platform }\end{array}$ \\
\hline$K_{1}^{\mathrm{a}}$ & 1.60 & 1.12 & 1.07 \\
$K_{2}^{\mathrm{a}}$ & 0.88 & 0.96 & 0.91 \\
$K_{3}^{\mathrm{a}}$ & 0.46 & 0.86 & 0.96 \\
$R^{\mathrm{b}}$ & 1.14 & 0.26 & 0.16 \\
\hline
\end{tabular}

${ }^{\mathrm{a}} K_{1}, K_{2}, K_{3}$ represent the average diameter of the printed fibers corresponding to the number $i$ level of various factors $(i=1,2,3) .{ }^{\mathrm{b}} R$ represents the range of the average of absolute value of difference.

mobile platform. And all these three parameters gives different influence on the diameter of the printed fibers. Because of the volume conservation, when the speed of stepper motor gets faster, the diameter gets bigger. And the faster the moving speed of nozzle is, the thinner the printed fibers are. The height from nozzle to platform acts as a disturbance to the printed fibers, and this disturbance's influence is unsetting. And some work of Yuk had a similarity of this part [20]. For the use of three different motors in controlling these three parameters respectively, the interaction of them was negligible.

During the experiment, a square ring $(50 \mathrm{~mm} \times 50 \mathrm{~mm})$ was printed, and the diameters of each edge were measured and recorded. The experiment was conducted at the room temperature of $23^{\circ} \mathrm{C}$, and all the rings were produced successively without a second feeding of pellets. Each ring was measured three times to ensure the reliability of the results. After getting the optimal parameter combinations, several cuboids $(10 \mathrm{~mm} \times 10 \mathrm{~mm})$ were printed to compare the quality of different parameter combinations.

2.4. Design of Experimental Table. Proper design of experiment helps the experiments and the results much more informative, Szekely et al. used a liquid chromatography tandem mass spectrometry (LC-MS/MS) method assisted optimization and robustness testing [26, 27]. Karakocak et al. used central composite design and response surface methodology in bioimaging [28]. And Toyota et al. made a process optimization for the application for liposomalization of FK506 [29]. The design of the experiment needs a concise process, an accurate result and an easy way to repeat the experiment. But the three factors discussed above would act as multiple influencing factors when carrying out practical experiments. The full factorial design and the fractional factorial design are two main approaches in multi-factor experiments. However it is hard to complete a full factorial design, especially when the sample of experiment is too big to save time or energy. As a fractional factorial experiment, orthogonal experimental design (OED) is widely used in multi-factor tests. Taking advantage of its orthogonality, some representative points are picked to represent the necessary sample of experiments among all the factorial experiments. Besides, OED provides a useful way to predict the full factorial experiments through analyses of the fractional experiments [30,31]. Using OED has three main advantages: (1) evenly distributing the data points, (2) reducing the number of experiments, (3) easily analyzing the experimental results with the contribution of range analysis and analysis of variance (ANOVA). Herein the OED is applied to optimize the process parameters.

As mentioned above, the major influencing factors that affect the diameter of the printed fibers in the extruding process are the speed of the stepper motor, the moving speed of nozzle, and the height from the nozzle to the platform. Considering 


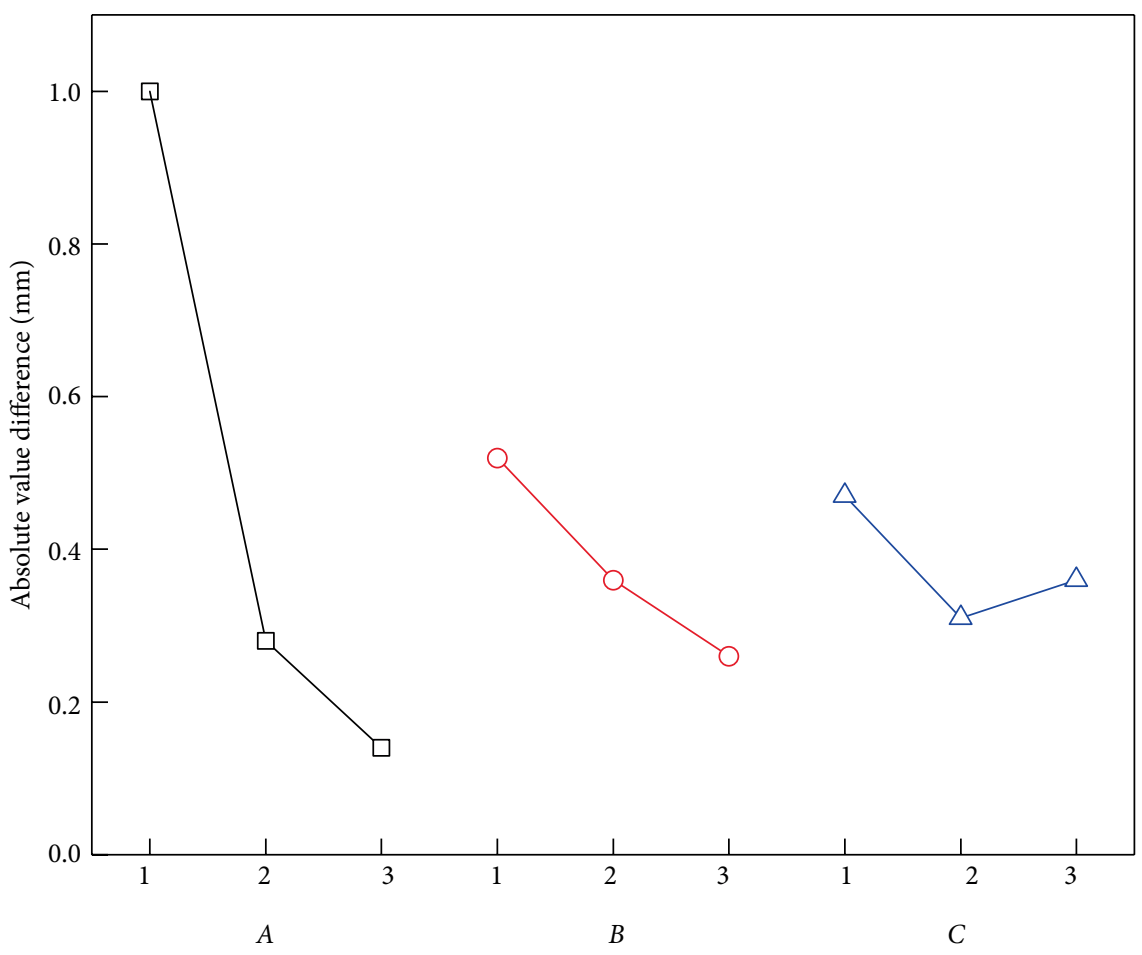

FIGURE 5: Relationship between the diameter and the three factors with three different levels (a) speed of the stepper motor; (b) moving speed of the nozzle; (c) height from the nozzle to the platform. The statistic was the absolute value of difference between the statistic in Table 5 and $0.6 \mathrm{~mm}$, the ideal diameter of the fiber.

TABLE 6: Analysis of variance for the orthogonal experiment.

\begin{tabular}{lccccc}
\hline Source & SS & DF & MS & $F^{\mathrm{a}}$ & $P$ \\
\hline$A$ & 1.97 & 2 & 0.99 & 49.25 & $0.01-0.05$ \\
$B$ & 0.10 & 2 & 0.05 & 2.50 & $>0.05$ \\
$C$ & 0.05 & 2 & 0.03 & 1.25 & $>0.05$ \\
Error & 0.04 & 2 & 0.02 & & \\
Corrected total & 2.16 & & & & \\
${ }^{\mathrm{a}} F_{0.05}(2,2)=9.0, F_{0.01}(2,2)=99.0$. & & &
\end{tabular}

the actual situation, each influencing factor consisted of three levels. Table 2 showed all of the three influencing factors and their levels. As well, the diameter of the printed fibers on the platform was selected to be the test index. And this experiment used a $\mathrm{L}_{9}\left(3^{4}\right)$ table. Table 3 showed the header design of the orthogonal experiment. Table 4 showed all of the factors of every experiment, while the blank column was set to access the errors according to the orthogonal design theory.

\section{Results and Discussion}

3.1. Die Swelling Phenomenon. Due to the polymer materials' viscoelastic, a die swelling phenomenon that some materials would become slightly bigger than the aperture of the nozzle while extruding appears [32]. The physical properties and extrusion pressure mainly explain this phenomenon. As shown in Figure 4, the rates of die swelling $\eta$ differed from each other because of the different speeds of the stepper motor.
For convenience, $\eta=1.2$ was chosen as the swelling rate in this study. When the printed fibers eventually attached to the platform, their idealized diameter would be $\eta$ times as large as the nozzle aperture. This parameter was chosen as a standard for the next calculation.

\subsection{Analysis and Optimization of Printing Parameters}

3.2.1. Range Analysis. After several experiments, the results were filled in Table 4. And a further calculation was done based on the results in Table 4 to get the optimal parameter combination. Moreover, according to the part above, $0.6 \mathrm{~mm}$ would be the ideal diameter when the aperture of the nozzle was $0.5 \mathrm{~mm}$. In the range analysis, $K_{1}, K_{2}$, and $K_{3}$ represented the average diameter of the printed fibers with the level number $i(i=1,2,3)$ to distinguish the various factors, while $R$ meant the range of values of $K_{1}, K_{2}$, and $K_{3}$ and was used to access the importance of each factor. The magnitude of $R$ determined the important degree of each factor - the larger the $R$ value was, the more important the factor was. These results have all been shown in Table 5. Relying on the $R$ values, the importance of factors with regard to the diameter followed this sequence: the speed of the stepper motor $>$ the moving speed of the nozzle $>$ the height from the nozzle to the platform. In Figure 5, the relationship between the diameter of the printed fibers and the three factors was shown in a more intuitive method. According to Figure 5, in selecting the lowest value, the best combination should be $A_{3} B_{3} C_{2}$ in order to obtain the minimum absolute value of difference. In other words, the optimal process condition was: a stepper motor speed 


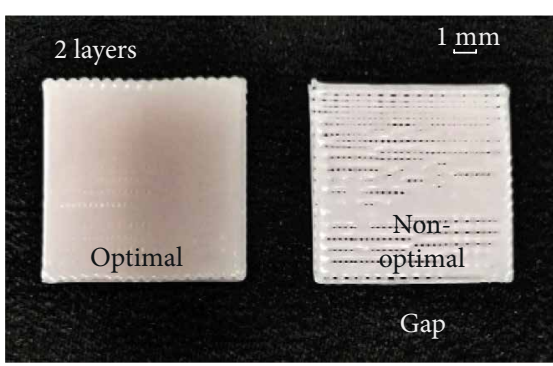

(a)

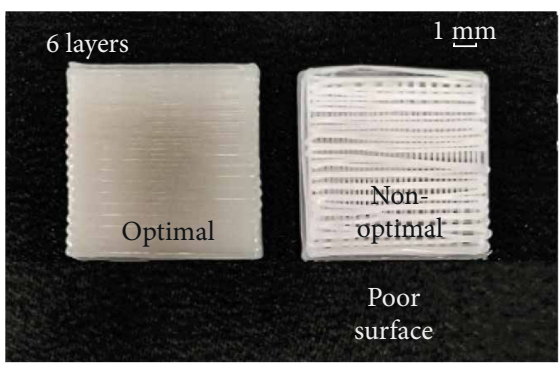

(c)



(b)

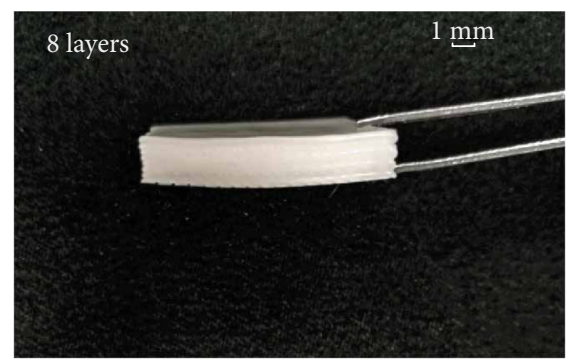

(d)

FIGURE 6: Cuboids with different combinations of parameters. The optimal parameter was $A_{3} B_{3} D_{2}$, while the nonoptimal parameter were: (a) $A_{3} B_{2} D_{3}$; (b) $A_{3} B_{3} D_{3}$; (c) $A_{3} B_{1} D_{1}$; and (d) $A_{3} B_{3} D_{2}$.

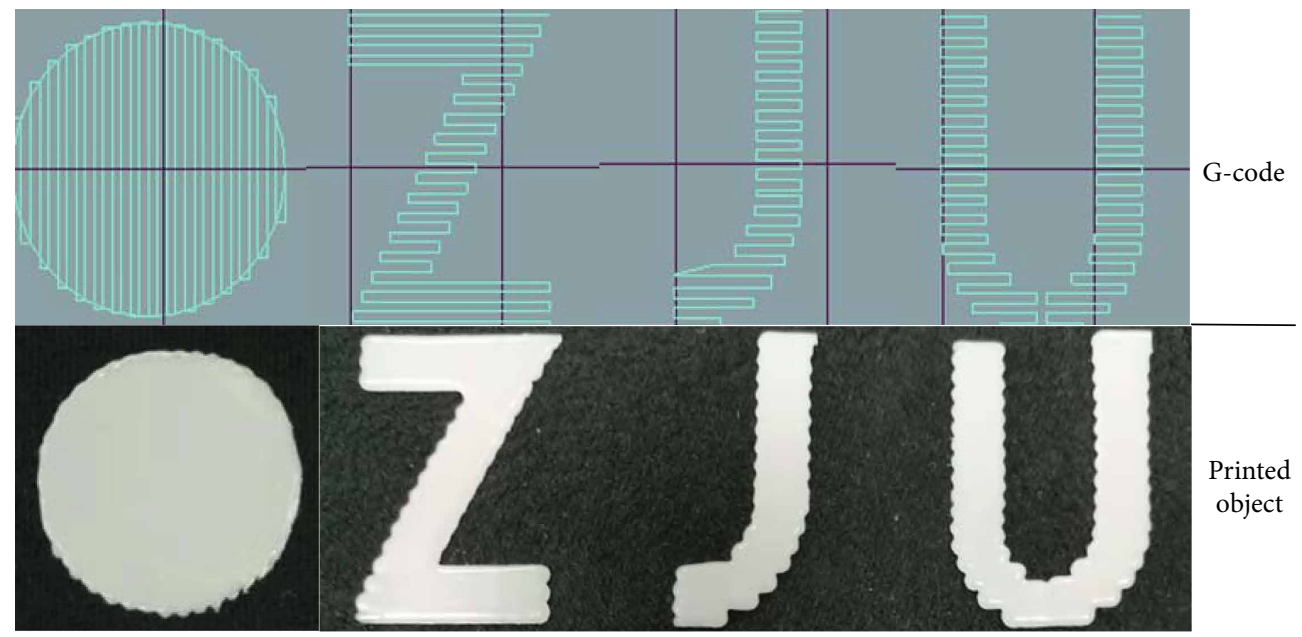

FIGURE 7: Other products with their appearance in G-code.

of $1.256 \mathrm{~mm}^{3} \mathrm{~s}^{-1}$, a nozzle moving speed of $9.6 \mathrm{~mm} \mathrm{~s}^{-1}$ and $1.1 \mathrm{~mm}$ height from the nozzle to the platform.

3.2.2. Analysis of Variance. ANOVA was applied to access the significance of the factors $[33,34]$. Table 6 showed the results of ANOVA by taking the diameter of the printed fibers as the observed index. As shown in Table 6, $P$-value, mean square error $(M S), F$-test statistic, the sum of square of deviations (SS), and degrees of freedom $(D F)$ made up the five essential values. The magnitude of the $P$ value compared to the given $\alpha$ significance level ( $\alpha=0.01$, and 0.05$)$ determined if each factor had a significant influence on the diameter of the printed fibers. The statistical significance level was set as follows: $*$ if $P<0.05, * *$ if $P<0.01$. $P_{A}$ was calculated within 0.01 to 0.05 , indicating that changing the factor $A$ had the most significant influence on the observed index. While $P_{B}$ and $P_{C}$ were bigger than 0.05 , which indicated that changing the factors $B$ and $C$ had a negligible effect on the observed index. In summary, ANOVA showed that the speed of the stepper motor had the most significant influence on the diameter of the printed fibers, the moving speed of the nozzle had only a small affect, and the height from the nozzle to the platform did not substantially affect the diameter.

3.3. Printing PCL Products. As discussed above, layer thickness, gap, outer-layer warping, and poor surface quality obviously influenced the visual appearance of the printed products. For example, the deeper the layer thickness is, the greater the possibility of overflow will be. And the gap inevitably leads to the inner and outer defects. Very much bigger or very 
much smaller diameter, by adjusting the combination of three influencing factors, could induce these defects. In Figures $6(a)-6(c)$, several cuboids with 2, 4, and 6 layers showed comparisons between the application of optimal parameters and the other parameters. Using the optimal parameters made products smoother and more regular. Then, when the printer was used to produce other shapes of structures, such as circular and letters, the appropriate diameter and proper circle interpolation algorithm made it easier to produce the products in Figure 7.

\section{Conclusion}

The novel 3D pellet printer developed in this study, made it convenient to use pellets in $3 \mathrm{D}$ printing compared to the traditional filament printer. The PCL pellets were directly extruded into different shapes by FDM technique. As for the process window, an orthogonal experiment was systematically conducted to select the optimal parameters of the speed of the stepper motor, the moving speed of the nozzle, and the height from the nozzle to the platform. Based on the results, the following conclusions can be drawn: (1) The 3D printer developed uses pellets as a raw material that have more potential rather than filaments in FDM 3D printing. (2) Due to the viscoelasticity of PCL, a die swelling phenomenon appears during the extruding period. Thus three important parameters are involved to adjust the diameter of the printed fibers. (3) According to the ANOVA, the speed of the stepper motor has the most significant effect on the diameter of the printed fibers ( $F=49.25$, while $P$ belongs to $0.1-0.5$ ), followed by the moving speed of the nozzle $(F=2.50$, while $P>0.5)$ and then the height from the nozzle to the platform $(F=1.25$, while $P>0.5)$. (4) Too much bigger or smaller of a diameter could cause defects like overflow, out-layer warping, gap, and poor surface quality. Eventually the optimal process condition was determined to be a stepper motor speed of $1.256 \mathrm{~mm}^{3} \mathrm{~s}^{-1}$, a nozzle moving speed of $9.6 \mathrm{~mm} \mathrm{~s}^{-1}$, and $1.1 \mathrm{~mm}$ height from the nozzle to the platform. In this condition, the products produced in the shape of cuboids, circles and letters are better than those produced with nonoptimal parameters. As a further prospect, this device could be applied to different kinds of materials and has the potential to make products quicker and more accurately. Some disadvantages like feeding difficulties and some problems about stability still exist. So for a next step plan, we will focus on improving the structure, the controlling of temperature and expanding the variety of materials like polypropylene (PP).

\section{Data Availability}

The data used to support the findings of this study are available from the corresponding author upon request.

\section{Conflicts of Interest}

The authors declare that they have no conflicts of interest.

\section{Acknowledgments}

The authors would like to acknowledge the financial support of the National Natural Science Foundation Council of China (No. 51875519 \& No. 51821093 \& No. 51635006), and the Zhejiang Provincial Natural Science Foundation of China (No. LZ18E050002).

\section{References}

[1] P. Zhao, H. B. Gu, H. Y. Mi, C. C. Rao, J. Z. Fu, and L. S. Turng, "Fabrication of scaffolds in tissue engineering: a review," Frontiers of Mechanical Engineering, vol. 13, no. 1, pp. 107-119, 2018.

[2] V. Secchi, R. Guizzardi, L. Russo et al., "Maltose conjugation to PCL: advanced structural characterization and preliminary biological properties," Journal of Molecular Structure, vol. 1159, pp. 74-78, 2018.

[3] D. H. Liu, W. Nie, D. J. Li et al., "3D printed PCL/SrHA scaffold for enhanced bone regeneration," Chemical Engineering Journal, vol. 362, pp. 269-279, 2019.

[4] S. Y. Kim, "Application of the three-dimensionally printed biodegradable polycaprolactone (PCL) mesh in repair of orbital wall fractures," Journal of Cranio-Maxillofacial Surgery, vol. 47, no. 7, pp. 1065-1071, 2019.

[5] B. M. Baker, A. O. Gee, R. B. Metter et al., "The potential to improve cell infiltration in composite fiber-aligned electrospun scaffolds by the selective removal of sacrificial fibers," Biomaterials, vol. 29, no. 15, pp. 2348-2358, 2008.

[6] H. Jeon, M. Lee, S. Yun et al., "Fabrication and characterization of 3D-printed biocomposite scaffolds based on PCL and silanated silica particles for bone tissue regeneration," Chemical Engineering Journal, vol. 360, pp. 519-530, 2019.

[7] Q. Gao, H. Gu, B. P. Zhao et al., "Fabrication of electrospun nanofibrous scaffolds with 3D controllable geometric shapes," Materials \& Design, vol. 157, pp. 159-169, 2018.

[8] C. Didaskalou, J. Kupai, L. Cseri et al., "Membrane-grafted asymmetric organocatalyst for an integrated synthesisseparation platform," ACS Catalysis, vol. 8, no. 8, pp. 7430-7438, 2018.

[9] W. G. Wang, B. Y. Huang, J. J. Byun, and P. Bartolo, "Assessment of PCL/carbon material scaffolds for bone regeneration," Journal of the Mechanical Behavior of Biomedical Materials, vol. 93, pp. 52-60, 2019.

[10] Y. Xiao, M. Zhou, M. Zhang, M. Liu, W. Y. Zhou, and M. D. Lang, "Hepatocyte culture on 3D porous scaffolds of PCL/ PMCL," Colloids and Surfaces B-Biointerfaces, vol. 173, pp. 185-193, 2019.

[11] W. Xue, Y. Hu, F. J. Wang, X. D. Yang, and L. Wang, " $\mathrm{Fe}_{3} \mathrm{O}_{4} /$ poly(caprolactone) (PCL) electrospun membranes as methylene blue catalyst with high recyclability," Colloids and Surfaces A: Physicochemical and Engineering Aspects, vol. 564, pp. 115-121, 2019.

[12] M. Ponjavic, M. Nikolic, S. Nikodinovic-Runic, J. Jeremic, S. Stevanovic, and S. Djonlagic J., "Degradation behaviour of PCL/PEO/PCL and PCL/PEO block copolymers under controlled hydrolytic, enzymatic and composting conditions," Polymer Testing, vol. 57, pp. 67-77, 2017.

[13] W. Y. Lin, H. Y. Shen, G. H. Xu, L. C. Zhang, J. Z. Fu, and X. L. Deng, "Single-layer temperature-adjusting transition method 
to improve the bond strength of 3D-printed PCL/PLA parts," Composites Part A-Applied Science and Manufacturing, vol. 115, pp. 22-30, 2018.

[14] Q. Gao, P. Zhao, R. Zhou, J. P. Wang, J. Z. Fu, and Y. He, "Rapid assembling organ prototypes with controllable cell-laden multiscale sheets. Bio-Design and," Manufacturing, vol. 2, no. 1, pp. 1-9, 2019.

[15] H. W. Kang, S. J. Lee, I. K. Ko, C. Kengla, J. J. Yoo, and A. Atala, "A 3D bioprinting system to produce human-scale tissue constructs with structural integrity," Nature Biotechnology, vol. 34, no. 3, pp. 312-319, 2016.

[16] P. Zhao, C. Rao, C. Gu, F. N. Sharmin, and J. Z. Fu, "Close-looped recycling of polylactic acid used in 3D printing: an experimental investigation and life cycle assessment," Journal of Cleaner Production, vol. 197, pp. 1046-1055, 2018.

[17] Y. Zhuang, W. Song, T. G. Ning et al., "3D-printing of materials with anisotropic heat distribution using conductive polylactic acid composites," Materials \& Design, vol. 126, pp. 135-140, 2017.

[18] C. A. Griffiths, J. Howarth, G. De Almeida-Rowbotham, A. Rees, and R. Kerton, "A design of experiments approach for the optimisation of energy and waste during the production of parts manufactured by 3D printing," Journal of Cleaner Production, vol. 139, pp. 74-85, 2016.

[19] O. A. Mohamed, S. H. Masood, and J. L. Bhowmik, “Optimization of fused deposition modeling process parameters: a review of current research and future prospects," Advances in Manufacturing, vol. 3, no. 1, pp. 42-53, 2015.

[20] H. Yuk and X. H. Zhao, "A new 3D printing strategy by harnessing deformation, instability, and fracture of viscoelastic inks," Advanced Materials, vol. 30, no. 6, Article ID 1870037, 2018.

[21] A. K. Sood, R. K. Ohdar, and S. S. Mahapatra, "Parametric appraisal of mechanical property of fused deposition modelling processed parts," Materials \& Design, vol. 31, no. 1, pp. 287-295, 2010.

[22] J. S. Chohan, R. Singh, K. S. Boparai, R. Penna, and F. Fraternali, "Dimensional accuracy analysis of coupled fused deposition modeling and vapour smoothing operations for biomedical applications," Composites Part B: Engineering, vol. 117, pp. 138-149, 2017.

[23] J. A. Covas and P. Costa, "A miniature extrusion line for small scale processing studies," Polymer Testing, vol. 23, no. 7, pp. 763-773, 2004.

[24] A. Goyanes, N. Allahham, S. Trenfield, J. Stoyanov, E. S. Gaisford, and A. W. Basit, "Direct powder extrusion 3D printing: fabrication of drug products using a novel singlestep process," International Journal of Pharmaceutics, vol. 567, Article ID 118471, 2019.

[25] S. Whyman, K. M. Arif, and J. Potgieter, "Design and development of an extrusion system for 3D printing biopolymer pellets," International Journal of Advanced Manufacturing Technology, vol. 96, no. 9-12, pp. 3417-3428, 2018.

[26] G. Szekely, B. Henriques, M. Gil, A. Ramos, and C. Alvarez, "Design of experiments as a tool for LC-MS/MS method development for the trace analysis of the potentially genotoxic 4-dimethylaminopyridine impurity in glucocorticoids," Journal of Pharmaceutical and Biomedical Analysis, vol. 70, pp. 251-258, 2012.

[27] G. Szekely, B. Henriques, M. Gil, and C. Alvarez, "Experimental design for the optimization and robustness testing of a liquid chromatography tandem mass spectrometry method for the trace analysis of the potentially genotoxic 1,3-diisopropylurea," Drug Testing and Analysis, vol. 6, no. 9, pp. 898-908, 2014.

[28] B. B. Karakocak, J. Liang, S. Kavadiya, M. Y. Berezin, P. Biswas, and N. Ravi, "Optimizing the synthesis of red-emissive nitrogendoped carbon dots for use in bioimaging," ACS Applied Nano Materials, vol. 1, no. 7, pp. 3682-3692, 2018.

[29] H. Toyota, T. Asai, and N. Oku, "Process optimization by use of design of experiments: application for liposomalization of FK506," European Journal of Pharmaceutical Sciences, vol. 102, pp. 196-202, 2017.

[30] P. Zhao, M. Y. Cao, H. B. Gu et al., "Research on the electrospun foaming process to fabricate three-dimensional tissue engineering scaffolds," Journal of Applied Polymer Science, vol. 135, no. 46, Article ID 46898, 2018.

[31] M. Y. Cao, F. Gu, C. C. Rao, J. Z. Fu, and P. Zhao, "Improving the electrospinning process of fabricating nanofibrous membranes to filter PM2.5," Science of the Total Environment, vol. 666, pp. 1011-1021, 2019.

[32] R. G. Larson and P. S. Desai, "Modeling the rheology of polymer melts and solutions," Annual Review of Fluid Mechanics, vol. 47, no. 1, pp. 47-65, 2015.

[33] W. F. Shan, L. M. Wu, N. Z. Tao, Y. W. Chen, and D. C. Guo, "Optimization method for green SrAl2O4:Eu2+, Dy3+ phosphors synthesized via co-precipitation route assisted by microwave irradiation using orthogonal experimental design," Ceramics International, vol. 41, no. 10, pp. 15034-15040, 2015.

[34] H. Salam, Y. Dong, I. J. Davies, and A. Pramanik, “Optimization of material formulation and processing parameters in relation to mechanical properties of bioepoxy/clay nanocomposites using Taguchi design of experiments," Journal of Applied Polymer Science, vol. 135, no. 5, Article ID 45769, 2018. 


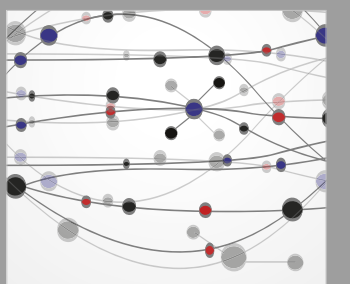

The Scientific World Journal
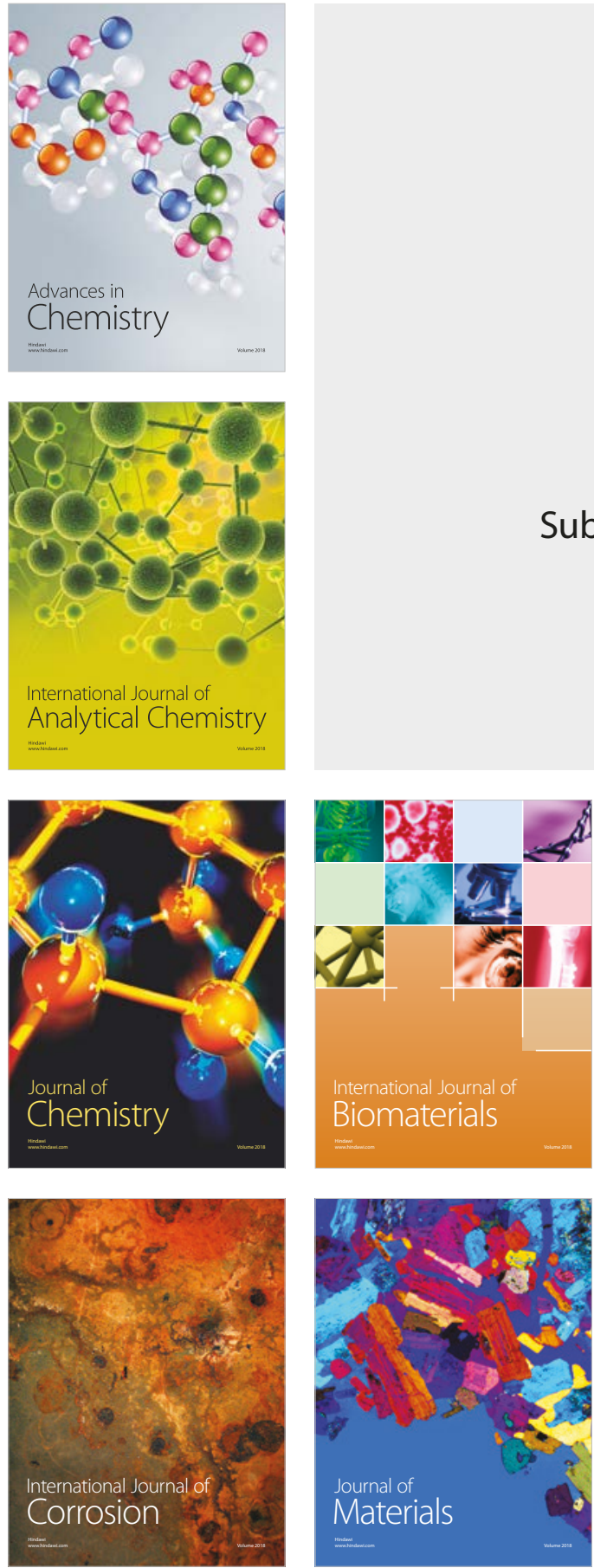

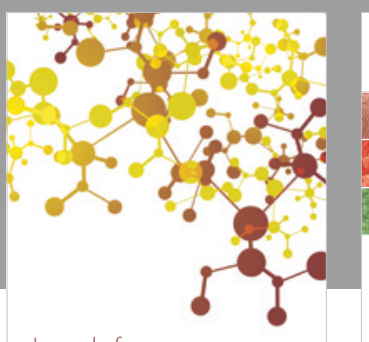

Journal of

Applied Chemistry
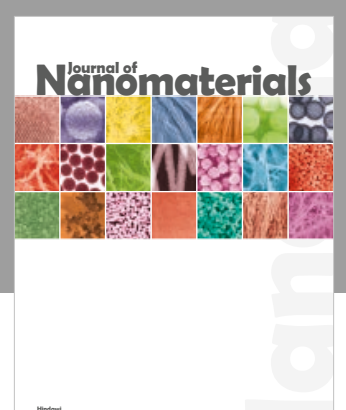

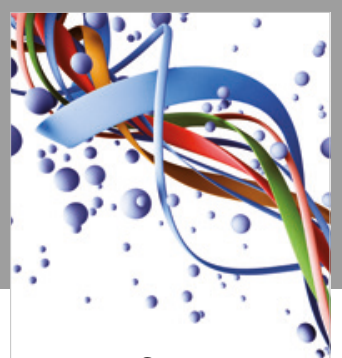

Scientifica

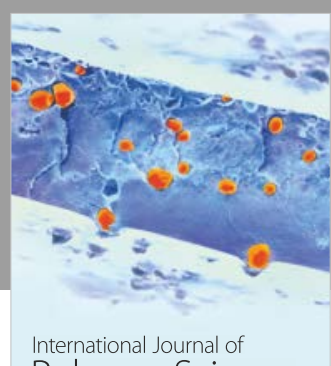

Polymer Science

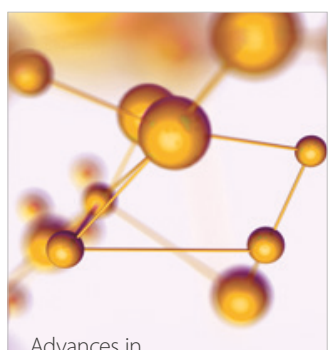

Physical Chemistry
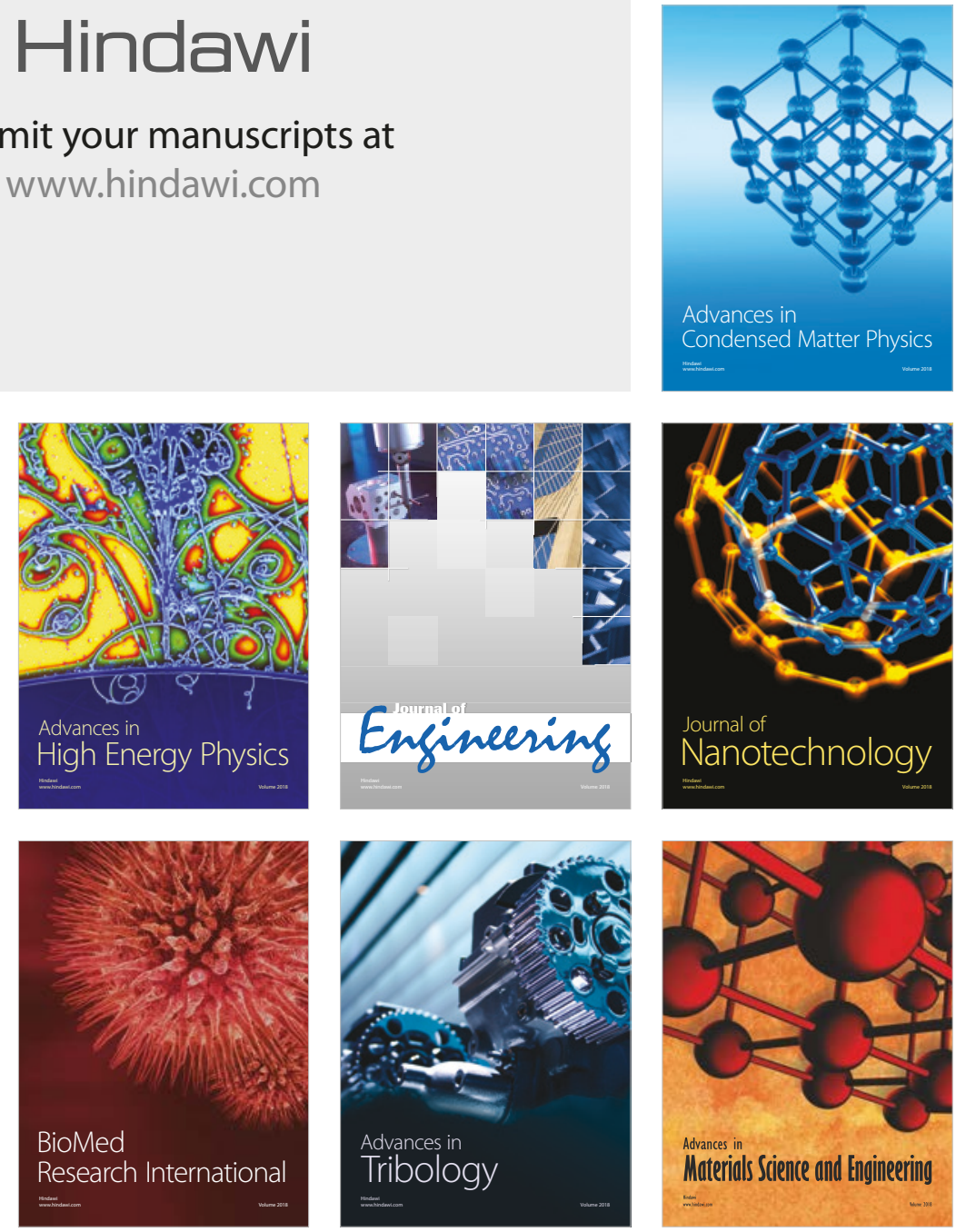